\title{
Measurement of Edge Plasma Heat Flux in Heliotron J Using a Thermal Probe
}

\author{
Hiroto MATSUURA, Ken NAKANO, Katsuyuki HOSAKA ${ }^{1)}$, Kenichi NAGAOKA ${ }^{2)}$, \\ Takashi MUTOH ${ }^{2)}$, Hiroyuki OKADA ${ }^{3)}$, Shinji KOBAYASHI ${ }^{3)}$, Tohru MIZUUCHI ${ }^{3)}$, \\ Katsumi $\mathrm{KONDO}^{2)}$ and Fumimichi SANO ${ }^{3)}$ \\ Graduate School of Engineering, Osaka Prefecture University, Osaka 599-8531, Japan \\ ${ }^{1)}$ Graduate School of Energy Science, Kyoto University, Uji 611-0011, Japan \\ ${ }^{2)}$ National Institute for Fusion Science, Gifu 509-5292, Japan \\ ${ }^{3)}$ Institute of Advanced Energy, Kyoto University, Uji 611-0011, Japan
}

(Received 8 January 2009 / Accepted 17 September 2009)

\begin{abstract}
Direct measurement of divertor heat flux is an important task. However, heat flux calibration is often difficult because heat diffusion in sensors takes longer than the discharge duration of present experiments. In this paper, using an unsteady heat conduction model, the heat flux in the Heliotron J edge plasma is measured for the first time. The obtained heat flux value, although it is time averaged, is positively correlated with plasma heating power, more precisely, with plasma internal energy. The angular distribution of heat flux around the probe axis shows a profile shape similar to previously reported probe current distributions.
\end{abstract}

(c) 2010 The Japan Society of Plasma Science and Nuclear Fusion Research

Keywords: Thermal probe, Divertor, Heliotron J, Heat flux, Heat conduction

DOI: $10.1585 /$ pfr.5.S1045

\section{Introduction}

It is well known that sheath regions exist between plasmas and solid plasma-facing components and that current through these sheath is determined by the sheath potential drop. According to sheath theory, the momentum and heat flux through the sheath are also functions of the sheath potential drop. Recently, a combined force Mach Langmuir probe [1] and a thermal probe [2,3] were proposed to measure these fluxes and to obtain not only electron parameters but also ion information, such as temperature. The first result of ion temperature measurement with a thermal probe were reported recently in [4], but the importance of the energy reflection coefficient in thermal probe measurement is noted in [5].

It is also very important to measure the heat flux itself in a divertor plasma. In the design of fusion reactors such as the International Tokamak Experimental Reactor (ITER), a vast heat flux $\left(>10\left[\mathrm{MW} / \mathrm{m}^{2}\right]\right)$ is expected to flow onto the divertor target plate through this sheath boundary. To check proposed methods for reducing this heat load, such as "detached plasma formation", direct measurement of heat flux is indispensable, since the relationship between the heat flux and plasma parameters is very complicated.

Conventionally, the simple formula $q=\gamma T_{\mathrm{e}} I_{\text {is }}$ has been used to estimate plasma heat flux $q$. However, even if the electron temperature $T_{\mathrm{e}}$ and ion saturation current $I_{\text {is }}$ can be measured, the sheath heat transmission factor $\gamma$

author'se-mail: matsu@me.osakafu-u.ac.jp can not be assigned exactly, since $\gamma$ depends not only upon plasma parameters such as space potential or ion temperature, but also upon probe tip surface processes such as energy reflection or secondary electron emission. In particular, since the ion temperature is larger than the electron temperature in divertor plasmas [6], the ion temperature contribution could not be ignored, as textbooks usually do. Thus, development of direct measurement tools for divertor heat flux is an important task. If simultaneous measurement of heat flux and edge plasma parameters yields enough data, experimental study of the heat transmission factor $\gamma$ would be possible in the future.

In this paper, the first results of heat flux measurement for the Heliotron $\mathrm{J}$ edge plasma are given. In section 2, the experimental setup is described. In section 3 , an unsteady heat conduction model is applied to thermal probe data in Heliotron J. Results are shown in section 4.

\section{Experimental Setup}

Heliotron $\mathrm{J}$ is a medium-sized helical-axis heliotron device with a helical winding coil of $L=1 / M=4$. Heliotron $\mathbf{J}$ is described in detail in Refs. [7] and [8]. Last year, the Hybrid Directional Probe (HDP) used in the Compact Helical System [9] was moved to Heliotron J under collaboration with the National Institute for Fusion Science (NIFS). Figure 1 shows a side view of the HDP. The HDP is composed of one magnetic probe sensor (Pin 6) and seven Langmuir probe tips (Pins 1-5, 7-8), five of which are equipped with type-K thermocouples (TC) and are also 


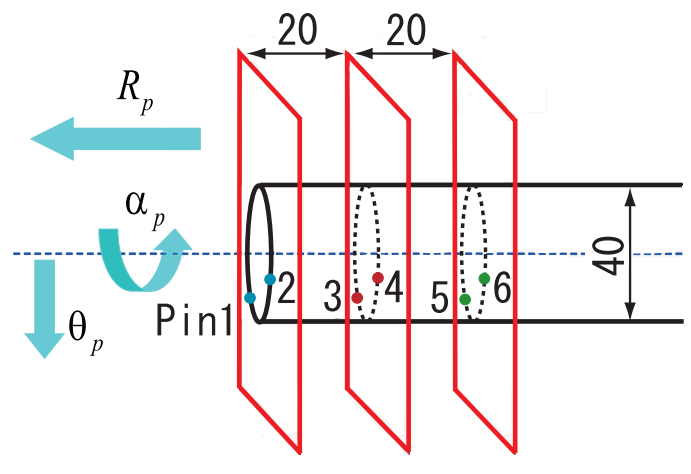

Fig. 1 Side view of the Hybrid Directional Probe (HDP) and its driving direction. Pin 7 and 8 are omitted for simplicity. The diameter of the cylindrical probe head is 40 [mm]. Pins 3 and 4 are set $20[\mathrm{~mm}]$ from the probe top (and also from Pins 1 and 2). Parameter $R_{\mathrm{p}}$ shows movement along the major radius. $\theta_{\mathrm{p}}$ is the poloidal rotation, and $\alpha_{\mathrm{p}}$ is the rotation around the probe head axis.

available as thermal probes. In Fig. 1, Pins 7 and 8 are omitted for simplicity. This paper mostly uses data from Pins 3 and 4. These pins are made of oxygen-free-copper, and their diameter and length are 4.0 and 10 [mm], respectively.

The HDP has a driving system of three parameters $\left(R_{\mathrm{p}}, \theta_{\mathrm{p}}, \alpha_{\mathrm{p}}\right)$ and its pin positions can be changed shot by shot. $R_{\mathrm{p}}$ is the HDP probe head shift along the major radius direction in units of $\mathrm{mm}$. When $R_{\mathrm{p}}$ increases, the HDP moves toward the confined main plasma (left side of Fig. 1). $\theta_{\mathrm{p}}$ is the swing angle in degrees along the poloidal direction, although in this paper only data for $\theta_{\mathrm{p}}=0$ are used. $\alpha_{\mathrm{p}}$ is the rotation angle in degrees around the axis of the cylindrical HDP head. In Fig. 1, intersection circles between the three planes and the cylindrical probe head shows the trajectory of probe pins during parameter $\alpha_{\mathrm{p}}$ scanning.

The HDP is installed at the port 7.5 cross section of Heliotron J, and the so called X-point can be studied. Figure 2 shows the outer part of the port 7.5 cross section and magnetic surface in the standard configuration. The horizontal solid line at $Z=0.271[\mathrm{~m}]$ is the trajectory of the HDP head axis for $\theta_{\mathrm{p}}=0$. When $R_{\mathrm{p}} \sim 135$, the top of the HDP head (also Pins 1 and 2) reaches the last closed flux surface(LCFS), although Pins 3 and 4 are still just outside the LCFS. Two symbols (cross and dagger) in this figure mark the positions of Pins 3 and 4, respectively, for $R_{\mathrm{p}}=210,185$, and 135. With changes in parameter $\alpha_{\mathrm{p}}$ within $R_{\mathrm{p}}=210$, Pin 3 ( and also Pin 4) moves along the vertical solid line in the lower figure at $R=1.36[\mathrm{~m}]$ and Pin 5 moves along the vertical solid line at $R=1.38[\mathrm{~m}]$.

Figure 3 shows the definition of the rotation angle $(\alpha)$ around the HDP axis. Looking from the inside of the vacuum chamber (left side of Fig. 1), the bottom position corresponds to $\alpha=0$, and $\alpha$ increases counterclock-
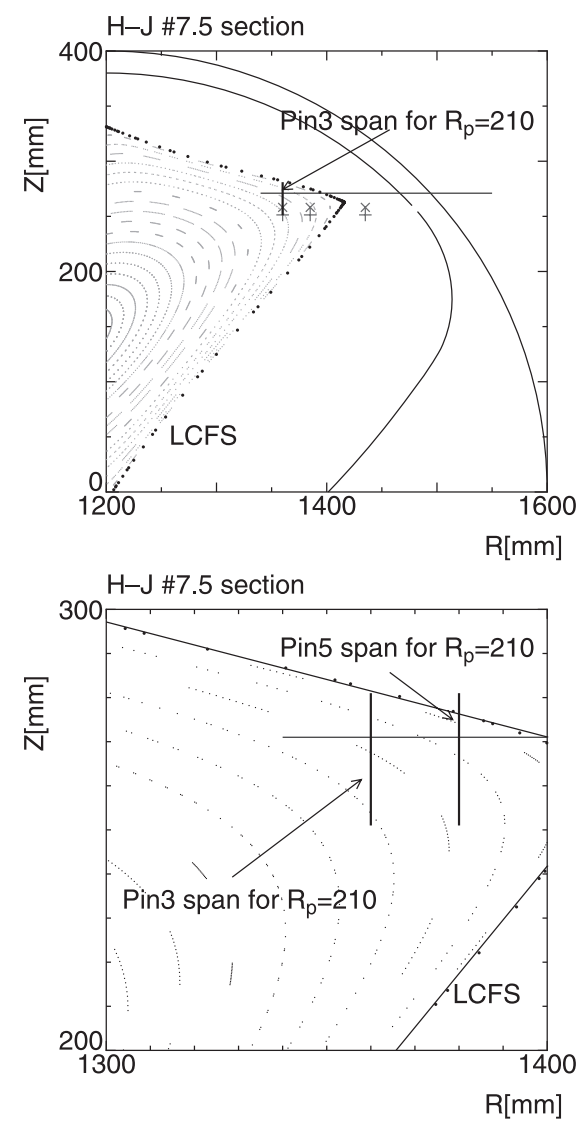

Fig. 2 Probe pins position of the Hybrid Directional Probe (HDP) on port 7.5 cross section. Lower figure is magnified to show the movement of Pins 3 and 5. HDP head moves along the horizontal solid line at $Z=0.271[\mathrm{~m}]$ with setting parameter $R_{\mathrm{p}}$. With changes in parameter $\alpha_{\mathrm{p}}$, Pin 3 (and also Pin 4) moves along the vertical solid line at $R=1.36[\mathrm{~m}]$, and Pin 5 moves along the vertical solid line at $R=1.38[\mathrm{~m}]$.
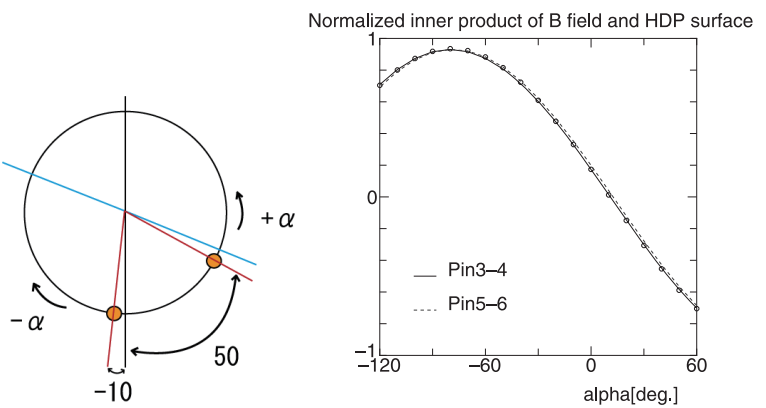

Fig. 3 Definition of rotation angle $\alpha$ around the HDP axis (left). From the inside of the vacuum chamber, the bottom position corresponds to $\alpha=0$, and $\alpha$ increases counterclockwise. The inner product of the normal vector of the HDP surface $(\vec{n})$ and magnetic field line direction $(\vec{B} /|B|)$ is also shown as a function of $\alpha$ (right).

wise. With changing driving parameter $\alpha_{\mathrm{p}}$, probe pins obtain data for different positions $\alpha$. The initial positions for $\alpha_{\mathrm{p}}=0$ are -10 [deg.] for Pin3 (and odd-numbered pins) and +50 [deg.] for Pin 4 (and even-numbered pins), as 
shown by small circles in Fig. 3. Due to mechanical limitations, only 120 [deg.] of rotation ( $\alpha_{\mathrm{p}}=-110 \sim+10$ ) are possible. So using two pairs of pins, data for $\alpha=-120 \sim$ +60 [deg.] could be obtained.

For different $\alpha$ positions, the angle between the magnetic field lines and probe surface varies. The inner product of the normal vector of the HDP surface $(\vec{n})$ and magnetic field line direction $(\vec{B} /|B|)$ is also shown in Fig. 3 as a function of $\alpha$. The solid line is for the Pin 3-4 section, and the dotted line is for Pins 5-6. The difference between the two lines is small, and both have a peak around $\alpha=-80$ [deg.]. Open circles in this figure also show $\vec{n} \cdot \vec{B} /|B|$. However, this $\vec{B}$ is calculated while neglecting the toroidal shift for $\alpha \neq 0$ [deg.]. A comparison of the open circles and solid line shows that the position shift of probe tips with $\alpha_{\mathrm{p}}$ scanning has little effect on the field line direction that the pins see.

\section{Heat Conduction Model}

The basic concept of the thermal probe is very simple. From probe tip temperature $\left(T_{\mathrm{p}}\right)$ data, heat flux to the probe surface $Q$ can be deduced by solving the heat conduction problem. For a DC discharge plasma, we can use the simple steady-state relation such that $Q$ is proportional to the steady-state tip temperature increment $\Delta T_{\mathrm{p}}$. However, heat flux calibration of the HDP thermal probes is not yet completed, mainly because the discharge pulse length ( $\Delta t \sim 0.1[\mathrm{~s}])$ is shorter than the thermal diffusion time in a probe tip (about $1[\mathrm{~s}]$ ), and a steady-state heat conduction model is not available.

Figure 4 shows an example of TC data measured at $\left(R_{\mathrm{p}}, \theta_{\mathrm{p}}, \alpha_{\mathrm{p}}\right)=(210,0,0)$ for a neutral beam injection $(\mathrm{NBI})$ plasma. The temperature increases almost immediately after the main discharge terminates and reaches a maximum value at about $t=0.5[\mathrm{~s}]$. After that, the TC signal shows an abnormal jump, which is thought to be due to helical coil current noise.

As a first step, we used a very simple model to analyze heat conduction in the probe pins. A probe pin is treated as semi-infinite plane, and plasma heat flux is treated as a delta-function-type short pulse. Then, the temperature in a probe pin is a function of time $t$ and distance from the pin surface $x$ and is given by

$$
\begin{aligned}
\Delta T & =T(x, t)-T_{\infty} \\
& =\frac{q \Delta t}{k} \sqrt{\frac{a}{\pi\left(t-t_{0}\right)}} \exp \left(-\frac{x^{2}}{4 a\left(t-t_{0}\right)}\right),
\end{aligned}
$$

where $k$ is the heat conductivity, $a$ is the thermal diffusivity, $q$ is the averaged heat flux density, $T_{\infty}$ is the initial temperature, and $t_{0}$ is the time when the heat pulse reaches the pin surface, which is indicated by a vertical line at $t=0.07$ [s] in Fig. 4.

For fixed $x$, the temperature response to the heat pulse shows a peak at $t=t_{0}+x^{2} / 2 a$. If the temperature increment reaches a maximum $\left(\Delta T=\Delta T_{\max }\right)$ at $t=t_{\max }$, the TC

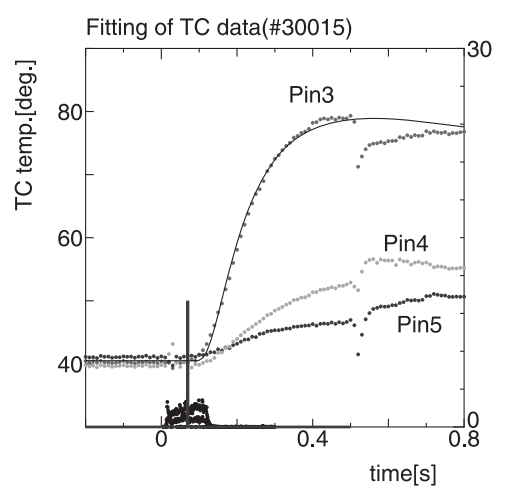

Fig. 4 Example of TC data and fitting for the HDP. Pin positions are $R=1.36[\mathrm{~m}]$ (Pins 3 and 4), $1.38[\mathrm{~m}]$ (Pin 5), and $Z=$ $0.25[\mathrm{~m}]$ (Pins 3 and 5), $0.26[\mathrm{~m}]$ (Pin4). The solid line fitting Pin 3 data is obtained by eq. (1) with $x_{\mathrm{tc}} \sim 1.07 \times$ $10^{-2}[\mathrm{~m}]$ and $q \Delta t \sim 2.9\left[\mathrm{~J} / \mathrm{mm}^{2}\right]$. To indicate the main plasma pulse length, the ion saturation currents of Pins 1 and 2 are also plotted in arbitrary units at the bottom.

sensor is expected to be located at $x_{\mathrm{tc}}=\sqrt{2 a\left(t_{\max }-t_{0}\right)}$. From the Pin 3 data in Fig. $4, x_{\mathrm{tc}} \sim 1.07 \times 10^{-2}$ [m]. The total heat that the probe pin receives $(q \Delta t)$ can be estimated by

$$
k \Delta T_{\max }=q \Delta t \sqrt{\frac{a}{\pi\left(t_{\max }-t_{0}\right)}} \exp \left(-\frac{1}{2}\right)
$$

If $q \Delta t \sim 2.9\left[\mathrm{~J} / \mathrm{mm}^{2}\right]$ is assumed, eq. (1) well reproduces the time evolution of the Pin 3 data in Fig. 4. Since the discharge pulse length $\Delta t$ of Fig. 4 can be estimated to be $\sim 0.12$ [s], Pin 3 is estimated to receive a heat flux of about $400[\mathrm{~W}]$ in the main discharge.

Note, however, that $x_{\mathrm{tc}}$ does not correspond exactly to the real position of TC connection point. The type-K TC used in the HDP has sheath material around the connection point that works to resist heat, and $t_{\max }-t_{0}$ may become longer than that expected from the true TC position.

According to eq. (1), if the TC position can be moved toward the probe surface or the TC sheath is removed, $x_{\mathrm{tc}}$ would become smaller, the TC signal response would be improved, and real time monitoring of heat flux might be possible. The TC signal of such an improved thermal probe would increase even during the main discharge pulse, which can not be fitted with eq. (1). So a second improved model for probe pin heat conduction is also developed. A probe pin is treated as a slab with effective thickness $L$. If probe pin cooling is strong enough, $L$ equals the length of a pin. Generally, however, a pin's heat transfer is weak, and $L$ is longer than the pin length. The plasma heat flux is treated as a box-type pulse,

$$
q(t)=\left\{\begin{array}{ll}
0 & \left(t<t_{0}=0, t>t_{1}\right) \\
q_{\infty} & \left(0<t<t_{1}\right)
\end{array} .\right.
$$


Then, the temperature in a probe pin is given by

$$
\Delta T(x, t)=\left\{\begin{array}{ll}
0 & \left(t<t_{0}=0\right) \\
\Delta T_{\infty}(x) & \\
+\Delta T_{1} A_{0} \sum_{n=0}^{\infty} A_{n} \cos \left(B_{n} \frac{x}{L}\right) \exp \left(-\tau_{n} \frac{t}{\tau}\right) & \left(0<t<t_{1}\right) \\
-\Delta T_{1} A_{0} \sum_{n=0}^{\infty} \tilde{A}_{n} \cos \left(B_{n} \frac{x}{L}\right) \exp \left(-\tau_{n} \frac{t-t_{1}}{\tau}\right) & \left(t>t_{1}\right)
\end{array} .\right.
$$

where $\tilde{A}_{n}=A_{n}\left(1-\exp \left(-\tau_{n} t_{1} / \tau\right)\right)$, and $\Delta T_{\infty}(x)=$ $-q_{\infty}(x-L) / \kappa$ is the steady state solution under a constant heat flux of $q_{\infty} . \Delta T_{1}=L q_{\infty} / \kappa, A_{0}=-8 / \pi^{2}, \tau=4 L^{2} / \pi^{2} a$ and other numerical coefficients are given by

$$
A_{n}=\frac{1}{(2 n+1)^{2}}, B_{n}=\frac{\pi(2 n+1)}{2}, \tau_{n}=(2 n+1)^{2} .
$$

By selecting $q$ (or $\Delta T_{1}$ ), $L, x_{\mathrm{tc}}$, and the pulse length $t_{1}-t_{0}$, the TC data of Fig. 4 can also be fitted with eq. (4), although it takes more time than fitting with eq. (1). For some plasma conditions, a finite temperature increment at the end of the main discharge pulse $\left[\Delta T\left(x_{\mathrm{tc}}, t_{1}\right)>0\right]$ is observed. However, for most experimental data, this increment is negligibly small, and the model of eq. (1) is sufficient for the present thermal probe data in Heliotron $\mathbf{J}$.

\section{Heat Flux Just the Inside LCFS}

Figure 5 shows the heat flux profile around the HDP head. A target plasma was produced by electron cyclotron heating $(\mathrm{ECH})$, and sustained by parallel neutral beam (NB) injection with only one source (BL-2). The horizontal axis is the angle around $\operatorname{HDP}(\alpha)$, and the vertical axis is the heat received during the entire discharge $(q \Delta t)$. $R_{\mathrm{p}}(=210)$ and $\theta_{\mathrm{p}}(=0)$ are fixed, and $\alpha_{\mathrm{p}}$ is scanned. Pin 3 (and Pin 5) covers $\alpha=-120 \sim 0$ [deg.], and Pin 4 covers $\alpha=-60 \sim 60$ [deg.]. For example, when $\alpha_{\mathrm{p}}$ is set to -50 , Pin 3 stays at $\alpha=-60$ [deg.], and Pin 4 detects the heat flux at $\alpha=0$ [deg.]. Although the data are limited and shows scattering, the maximum heat flux is found around $\alpha=-30$ [deg.] for Pins 3-4. As shown in Fig. 1, Pin 5 circulates on another plane and shows a rather wider heat flux profile with peak at $\alpha=-60$ [deg.]. If these heat flux profiles are determined by the projection of the pin's surface area, it is expected from Fig. 3 that the heat flux reaches a maximum at $\alpha=-80$ [deg.] and is zero at $\alpha=0$ [deg.] Data from Pins 3-4 data contradicts this expectation. A similar result has already been reported in [10]. Even if the field lines are nearly parallel to the probe pin surface, the sheath potential attracts plasma ions, and finite heat flux reaches the probe pin, since probe pins are usually biased negatively.

As shown by the small vertical line at $R=1.36[\mathrm{~m}]$ in Fig. 2, probe pins reach the LCFS at $\alpha=-120$ [deg.] (mechanical limit), and they go most deeply into the main

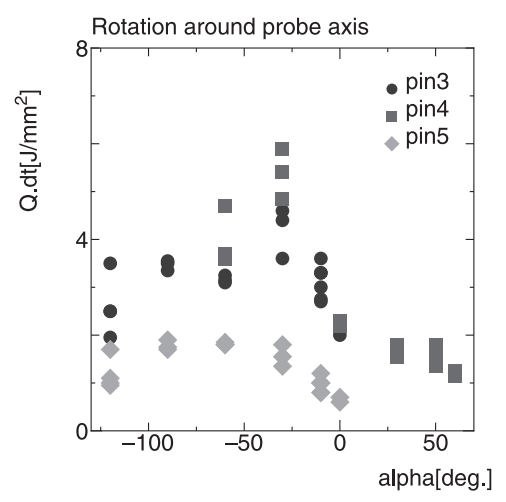

Fig. 5 Heat flux profile around the HDP head. Horizontal axis is the angle around the HDP $(\alpha)$; vertical axis is the heat received during the entire discharge $(q \Delta t)$. For rotation angle $\alpha_{\mathrm{p}}=0$, Pin 3 (and Pin 5) is at $\alpha=-10$ [deg.], and Pin 4 is at $\alpha=50$ [deg.].

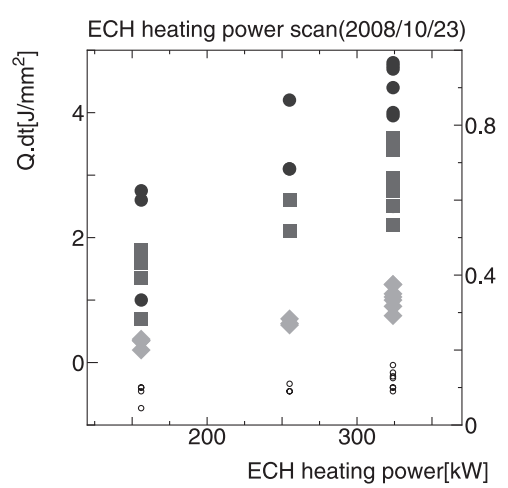

Fig. 6 ECH power scan effect on heat flux. Data symbols are the same as in Fig. 5. Small open circles are also plotted for diamag monitor values (right axis, arbitrary units).

plasma at $\alpha=0$ [deg.]. Thus, if the gradient of plasma parameters (density, temperature, potential, etc.) is significant, heat flux is expected to show a maximum at $\alpha=0$ [deg.] and a minimum at $\alpha=-120$ [deg.]. However, this expectation also does not agree with Fig. 5. Similar profiles have already been obtained for ion saturation current measurement. A possible explanation for these profiles is the existence of plasma flow directed toward the $\alpha=-50$ [deg.] or $\alpha=130$ [deg.] direction. If this hypothesis is true, the angular profile must have a periodicity of 180 [deg.]. Unfortunately, the present heat flux data does not confirm a clear minimum value around $\alpha=40$ [deg.]. Further measurement for different NBI beam lines and different magnetic configurations is now being planed.

Figure 6 shows the change in heat flux with plasma heating power. (In this case, only ECH was used to heat the the plasma.) Data symbols are the same as in Fig. 5 (Pin 3: circles, Pin 4: squares, Pin 5: diamonds). $R_{\mathrm{p}}$ and $\theta_{\mathrm{p}}$ are also the same as in Fig. 5 and $\alpha_{\mathrm{p}}$ is kept at 0 , which means that the rotation angle of Pin 3 (and Pin 5) is $\alpha=-10$ [deg.] and that of Pin 4 is $\alpha=50$ [deg.]. With increasing plasma 


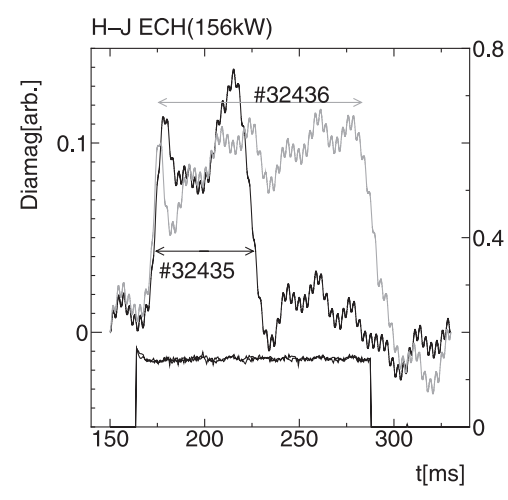

Fig. 7 Diamag monitor signal for two successive shot with the same ECH power and slightly different gas puffing control.

heating power, the estimated heat flux for each pins also increases. However, data scattering is rather large, because keeping the line-averaged density nearly constant for different ECH heating powers is difficult. For high power $\mathrm{ECH}$, strong gas puffing is necessary to overcome so-called density clamping. When the ECH power is reduced, extra gas puffing sometimes terminates the main discharge. One example is given in Fig. 7. The ECH power of shots 32435 and 32436 is the same (about $156[\mathrm{~kW}]$ ), but the discharge of \#32435 terminates during the $\mathrm{ECH}$ heating pulse, and the discharge time is only $40 \%$ of that of \#32436, while the stored energy is almost the same around $t=200[\mathrm{~ms}]$.

The $q \Delta t$ data for \#32435 in Fig. 6 are also much smaller than for \#32436. For Pin 3, $q \Delta t$ is about $1.0\left[\mathrm{~J} / \mathrm{mm}^{2}\right](\# 32435)$ and $2.6\left[\mathrm{~J} / \mathrm{mm}^{2}\right](\# 32435)$. Considering that the discharge time $\Delta t$ for \#32435 is much shorter than the ECH heating pulse length, the averaged heat flux is nearly equal for these two shots. Thus, to study the relationship between heating power and heat flux measured with the thermal probe method and the present heat conduction model, knowledge of the real discharge time would be necessary. On the other hand, although the present method of measuring heat flux can not obtain its time variation, it could be used as a monitoring tool to watch shot reproducibility to supplement ion saturation current. If the probe position or bias voltage is kept constant and the TC signal (or estimated heat flux) after a shot changes, we can see that something wrong has occurred in the shot.

\section{Summary}

The results obtained in this paper are summarized as follows.

- Heat conduction models to calibrate heat flux detected with the HDP are constructed.

- Using TC evolution data, the averaged heat flux in the Heliotron $\mathbf{J}$ edge plasma is estimated for the first time; no special modeling of sheath heat transmission factor $\gamma$ is necessary.

- By rotating the HDP around its axis, the heat flux angular profile is measured. Although the edge plasma profile effect must be estimated carefully in future studies, the obtained heat flux profile might be due to some kind of plasma flow.

- With increasing plasma heating power, the estimated heat flux also seems to increase, but knowledge of the real discharge time is necessary to estimate the heat flux exactly.

To monitor heat flux during the main plasma discharge, improved TC response is necessary. One method is to reduce the heat resistance between the $\mathrm{TC}$ and the probe pins by removing the TC's sheath material. Another is to move the connecting points of the TC toward the pin surface, where plasma irradiation occurs. Design and construction of a new thermal probe considering these improvements are left as future work.

\section{Acknowledgements}

The authors are grateful to the Heliotron J supporting group for their excellent arrangement of experiments. One of the authors (H. M.) acknowledges Prof. Y. Nakamura and Dr. S. Yamamoto of Kyoto University for their advice on the Heliotron $\mathrm{J}$ magnetic configuration and data acquisition system. This work is performed partially with the support and under the auspices of the NIFS Collaborative Research Program (NIFS08KUHL021).

[1] T. Lunt et al., Proc. 32nd EPS Conf. on Plasma Phys., P1005, Tarragona, Spain (2005).

[2] E. Stamate et al., Appl. Phys. Lett. 80, 3066 (2002).

[3] H. Matsuura et al., Contrib. Plasma Phys. 46, 406 (2006).

[4] K. Kurihara et al., Plasma Fusion Res. 2, S1082 (2007).

[5] H. Matsuura et al., Proc. 2nd IFHT., Tokyo, Japan 93 (2004).

[6] M. Kočan et al., Plasma Phys. Control. Fusion. 50, 125009 (2008).

[7] T. Obiki et al., Nucl. Fusion 41, 833 (2001).

[8] F. Sano et al., Nucl. Fusion 45, 1557 (2005).

[9] K. Nagaoka et al., Plasma Fusion Res. 2, S1092 (2007).

[10] Koch et al., J. Nucl. Mater. 337-339, 211 (2005). 